\title{
Review: aromatherapy massage is associated with small, transient reductions in anxiety
}

Cooke B, Ernst E. Aromatherapy: a systematic review. Br J Gen Pract 2000 Jun;50:493-6.

\section{QUESTION: Does aromatherapy (treatment with aromatic plant extracts known as essential oils) have an effect on clinical outcomes in patients with various conditions?}

\section{Data sources}

Clinical trials that were published in any language up to June 1999 were identified by searching Medline, EMBASE/Excerpta Medica, British Nursing Index, CISCOM, and AMED using the terms alternative medicine, massage, essential oils, and aromatherapy; and by contacting experts.

\section{Study selection}

Randomised controlled trials of the use of aromatherapy in human patients were included. Studies of the local effects of aromatherapy (eg, antiseptic effects of tea tree oil) and pre-clinical studies of healthy volunteers were excluded.

\section{Data extraction}

Data were extracted on the condition under investigation, sample characteristics, type of intervention and placebo, outcomes, and results. Methodological quality of studies was assessed using the Jadad scale (randomisation, blinding, and accounting for dropouts).

\section{Main results}

12 studies were identified. 6 trials evaluated the effects of aromatherapy massage on anxiety or wellbeing in patients with cancer, patients who had cardiac surgery, and patients in an intensive care unit. All interventions were given by nurses in hospital settings. Jadad scores for methodological quality ranged from $0-2$ out of 5 , with a rating of 5 indicating the highest quality. Study results could not be pooled because of study heterogeneity. 5 of the studies reported that patients who received aromatherapy massage had small reductions in anxiety or improvements in wellbeing immediately after the intervention: foot massage with plain oil plus orange blossom oil; massage with oil plus aroma; massage plus lavender oil; and full body massage with bland oil plus chamomile oil (2 studies). 1 study of massage with almond oil plus English lavender oil compared with spike lavender oil found no group differences for anxiety.

The remaining 6 trials each dealt with the effects of a unique intervention on a specific condition. 5 of the trials found a benefit of aromatherapy over placebo or control: inhalation of a vaporised aromatic mixture to improve pulmonary function in the common cold; oral aromatic liquid to prevent relapse of bronchitis; dummy cigarettes with black pepper smell or menthol smell for smoking withdrawal symptoms; inhalation of steam plus aroma for anxiety; and scalp massage of carrier oils plus essential oils for alopecia areata. 1 study found no difference for bath water with natural or synthetic lavender oil and placebo for perineal discomfort after childbirth.

\section{Conclusions}

Aromatherapy massage has a small, transient effect on reduction of anxiety immediately after administration. Individual studies suggest possible benefits of inhaled or oral aromatherapy for various conditions.

\section{COMMENTARY}

This systematic review by Cooke and Ernst is particularly timely as aromatherapy, and particularly aromatherapy massage, are increasingly used by nurses working in areas such as cancer and palliative care. The review will be of interest to clinicians involved in these and other areas of health care where complementary treatment services are being considered. This is one of the first rigorous evaluations of the evidence surrounding the use of aromatherapy.

The authors suffered the fate of many systematic reviewers in that they found only a few trials, which were of variable quality and involved small numbers of patients. Their searching and inclusion criteria are presented clearly, as are details of the studies identified and included. Of the 12 trials identified, they chose to concentrate on the 6 trials of aromatherapy massage; the remaining 6 studies were unique comparisons of different aromatherapy interventions.

The authors clearly report the methodological quality of the trials, which allows the reader to judge the results of each study included. It was not possible to use meta-analytic techniques to combine the individual studies to obtain an overall estimate of effect because the studies were heterogeneous and used different outcome measures.

The conclusion drawn is that aromatherapy massage reduces anxiety to some extent. The authors state that the anxiety reduction associated with aromatherapy massage is transient, but they do not report the data which describe the duration of effect. Furthermore, uncertainty exists as to the most appropriate outcome measures for complementary treatment evaluation. ${ }^{1}$

Cooke and Ernst's discussion raises some important issues about the use of aromatherapy massage within the UK National Health Service and elsewhere. The key question is whether, in the absence of good evidence of more than transient effects, we can regard aromatherapy massage as a healthcare intervention that should be funded accordingly, or as something pleasant and relaxing that may be delivered by informal carers or paid for as a separate service by patients or charities.

Kate Flemming, RGN, MSc Lecturer, Centre for Evidence Based Nursing University of York York, UK

1 Botting D, Cook R. Evaluating the effectiveness of complementary therapies. International Journal of Palliative Nursing 1998;4:32-6. 